\title{
Observational extinction: Observation of nonreinforced responding reduces resistance to extinction in rats
}

\author{
C. M. HEYES and E. JALDOW \\ University College London, London, England \\ and \\ G. R. DAWSON \\ Merck, Sharp \& Dohme Neuroscience Research Centre, Harlow, England
}

\begin{abstract}
Rats trained to push a joystick to the left or right for food reward were given two successive tests in which neither response was reinforced. Prior to Test 1 , subjects were either confined in the apparatus with a passive conspecific (Group None), or allowed to observe a conspecific demonstrator making 50 nonreinforced responses in the direction that had been rewarded during observer training (Group Same) or in the opposite direction (Group Different). In Test 1, Group Same made fewer previously reinforced responses than did Group Different, which made fewer than Group None, and Groups Same and Different each made fewer previously nonreinforced responses than did Group None. In Test 2, Group Same made fewer previously reinforced responses than did Group None. These results indicate that observation of nonreinforced responding can reduce resistance to extinction (Test 1 ) and spontaneous recovery (Test 2) in rats.
\end{abstract}

Most attempts to demonstrate that nonhuman animals can acquire a response through observation of reinforced responding (a phenomenon known as "imitation" or "observational learning"') have had ambiguous results (Galef, 1988; Heyes, in press). Typically, they show that rats which have observed a conspecific demonstrator making reinforced responses subsequently acquire the same response faster than controls that have been exposed to a demonstrator receiving noncontingent reward, or to a passive conspecific, before testing (e.g., Levine \& Zentall, 1974; Zentall \& Levine, 1972). Such effects may be due to response learning by observation, but they may also be examples of local enhancement (Thorpe, 1956) or observational autoshaping (Hogan, 1986, 1988) in which the behavior of a conspecific directs the observer's attention to the manipulandum and thereby affects frequency of contact with the manipulandum on test, either directly, or via stimulus-reinforcer learning during observation. However, relatively clear evidence of response learning by observation has been provided in at least two recent experiments with procedures in which naive animals observed trained conspecifics acting on a single manipulandum in one of two different ways (Galef,

\footnotetext{
This research was supported by a grant from the Leverhulme Trust. We are grateful to Paula Durlach for her suggestions regarding the design of the experiment, to Roger Bunce and Jim Chambers for help in the design and construction of the apparatus, and to Henry Plotkin, Phil Reed, and an anonymous reviewer for comments on an earlier draft of the manuscript. Correspondence should be addressed to $\mathrm{C}$. M. Heyes, Department of Psychology, University College London, Gower Street, London WC1E 6BT, England.
}

Manzig, \& Field, 1986; Heyes \& Dawson, 1990). Galef et al. found that budgerigars that had observed a conspecific using its feet to lift a flat cover from the top of a food cup were subsequently more likely to use their feet to lift the cover than birds that had observed a conspecific using its beak. This effect was apparent only in performance on the first two test trials.

Heyes and Dawson (1990) used a "bidirectional control" procedure, in which magazine-trained rats were allowed to observe a conspecific pushing a joystick to the left or right with continuous reinforcement, before they were given access to the joystick for the first time and rewarded for both left and right responses. On test, the group that had observed left pushing made a greater proportion of their responses to the left than did the group that had observed right pushing. The observer and demonstrator rats were face to face during the observation phase of the experiment, and the observers were given access to the joystick from the position previously occupied by the demonstrators. This arrangement made it unlikely that the effect reported by Heyes and Dawson (1990) was due to stimulus-reinforcer learning during observation, and subsequent experiments have apparently confirmed that demonstrator-consistent responding in the bidirectional control procedure is due to response learning by observation. In one of these experiments (Heyes, Dawson, \& Nokes, 1992), observer rats pushed the joystick in the same direction, relative to their own bodies, as had the demonstrators, even when this action resulted in the joystick moving toward a different location in space. The other experiment (Heyes, Jaldow, Nokes, \& Dawson, 1993) showed that observers of right and left joystick 
movement did not differ in their directional preferences when they had observed the joystick moving automatically, rather than through the action of other rats.

Although the foregoing experiments had shown that observation of reinforced responding could affect response acquisition in rats, in the present experiment we examined the effects of observation of nonreinforced responding on response extinction. In several studies, researchers have sought evidence that observation of reinforced responding can affect extinction performance (Henning \& Zentall, 1981; Heyes \& Dawson, 1990; Roder, Timmermans, \& Vossen, 1989), but in none of them, as far as we are aware, were demonstrators observed, or observers tested, in extinction.

In the present experiment, each rat first received conventional (nonobservational) training in which it was rewarded for left or right joystick pushing, followed by two successive extinction tests in which neither left nor right responses were reinforced. Immediately prior to the first test, subjects were confined in the apparatus either with a passive conspecific (Group None), or with a conspecific demonstrator making 50 nonreinforced responses either in the direction that had been rewarded during observer training (Group Same) or in the opposite direction (Group Different). Test performance was measured in terms of the number of responses that the observers made in the direction that had been reinforced during observer training (previously reinforced responses), and in the direction that had not been reinforced during observer training (previously nonreinforced responses).

\section{METHOD}

\begin{abstract}
Subjects
Forty experimentally naive, male hooded Lister rats, obtained from Charles Rivers (U.K.), were approximately 6 months old when they served as subjects. Sixteen of these rats were demonstrators, and the remaining 24 were observers. The subjects were kept in groups of 5 ( 2 demonstrators and 3 observers) in molded plastic and metal hanging cages $(54 \times 32 \times 21 \mathrm{~cm})$, with free access to water. All were maintained at $85 \%$ of their free-feeding body weights throughout the experiment.
\end{abstract}

\section{Apparatus}

The animals were trained and tested in four identical operant chambers, each measuring $50 \times 25 \times 20 \mathrm{~cm}$. The walls of each chamber were made of sheet metal; the ceiling, of clear Perspex; and the floor, of a metal grid. Each chamber was divided into two compartments of equal size by a $1-\mathrm{cm}$-gauge wire-mesh partition. In the compartment used for demonstrations and testing, an aluminum alloy joystick $(0.6 \mathrm{~cm}$ in diameter) was suspended from the ceiling, half way between the side walls. The free end of the joystick, which was $6.5 \mathrm{~cm}$ above the floor when the joystick was in a vertical position, could be moved only to the left or right in a plane parallel to that of the partition. The joystick was separated from the partition by a distance of $4 \mathrm{~cm}$. This distance was chosen because it was great enough to prevent an observer rat from reaching through the partition and contacting the joystick during observation, and short enough to prevent an animal in the demonstration/test compartment from manipulating the joystick from the partition side. The latter feature ensured that when observers were responding on test, they were facing in the direction opposite to that from which they had viewed the joystick during observation training.

Movement of the joystick by the demonstrators and observers was recorded via a low-torque potentiometer pivot at the top of the joystick, above the roof of the chamber containing the animals. A constant voltage was applied across the pivot. Movement of the joystick by a rat resulted in rotation of a brush within the potentiometer, and hence in a voltage proportional to the degree of joystick displacement at the brush terminal. This dc potential was converted to a dig ital signal read by a BBC Master computer running Spider (Paul Fray Ltd.) on-line control language. During observation training and testing, the demonstrators and observers had to displace the free end of the joystick $7.5 \mathrm{~cm}$ to register a response. After displacement, the joystick was assisted back to its vertical starting position by a weight and a spring resting on the pivot of the manipulandum.

In addition to the joystick, the demonstration/test compartment contained a food tray situated at floor level in the middle of the wall opposite the partition. The food tray was illuminated on the inside by a 24-V, 2.8-W bulb, and entries to the tray were recorded via a photocell beam. Whenever a subject made a response designated for reinforcement, a 45-mg food pellet of mixed composition was automatically delivered to the food tray. Operation of the magazine was accompanied by the delivery of a $1000-\mathrm{Hz}, 90-\mathrm{dB}$ tone of 0.1 -sec duration from a loudspeaker directly above the food tray, extinguishing of the house light, and illumination of the tray light. The house light was switched on, and the tray light was switched off, $5 \mathrm{sec}$ later, or, if a tray entry was made within $5 \mathrm{sec}$, when the next tray entry was registered. Following the delivery of a food pellet, the animal had to enter the tray before another reinforcer became available. Responses that were not designated for reinforcement had no programmed consequences.

\section{Procedure}

Each session began with illumination of the house light and ended, after 50 food pellets had been delivered (unless otherwise stated), when that light was extinguished. A response was scored as "left" if it resulted in a displacement of the joystick toward the near side of the operant chamber, and as "right" if it resulted in displacement of the joystick in the opposite direction. As observers and demonstrators faced one another on opposite sides of the partition and of the joystick, when a demonstrator made a "right" response, the joystick moved to the left in the demonstrator's visual field and to the right in the observer's visual field.

Demonstrator training. Initially, each demonstrator received four daily sessions of magazine training in the demonstration/test compartment, from which the joystick had been removed. Thirty food pellets were delivered on a random time 45 -sec schedule in each session. The demonstrators were then trained, in 13-17 daily sessions, to push the joystick to the left $(n=8)$ or to the right $(n=8)$. During the first session, both left and right responses were continuously reinforced, and a joystick displacement of $2.5 \mathrm{~cm}$ was sufficient to register a response. Over the next four sessions, in which either right or left responding was continuously reinforced, the extent of joystick displacement necessary to register a response was increased gradually until, from Session 5 onward, displacements of $7.5 \mathrm{~cm}$ or more were required. Discriminative responding was rewarded on a variable interval 15-sec (VI 15) schedule in Sessions 6 and 7, and on a VI30 schedule during all subsequent sessions. To allow demonstrators to become accustomed to being observed while pushing the joystick, a "dummy observer" was placed in the observation compartment during each demonstrator's final three sessions of training. The dummy rats, which were about the same age as the demonstrators, had received instrumental training in the apparatus as part of a previous experiment.

Observer training and testing. The observers were magazine trained, and pretrained to push the joystick to the left $(n=12)$ or 
right ( $n=12$ ) with continuous reinforcement, in the same way as were the demonstrators. Observation training and testing occurred the day after the observers' fourth and final session of conventional instrumental pretraining.

Each observer was assigned, before the experiment began, to one of three groups for observation training: Same $(n=8)$, Different $(n=8)$, and None $(n=8)$. Half the observers in each of these groups were pretrained to push the joystick to the left, and half to push it to the right. On the test day, each rat in Group Same was placed in the observation compartment and allowed to observe a demonstrator pushing the joystick 50 times, with no programmed consequences, in the direction that had been reinforced during that observer's pretraining. When the demonstrator had completed its last response, it was removed from the apparatus, and the observer was immediately transferred to the demonstrator/test compartment for its first extinction test, in which neither left nor right joystick responses had programmed consequences. This test terminated, with the house light being switched off and the observer returned to its home cage, when the observer had reached a criterion of 5 min without responding. Thirty minutes later, the observer was returned to the demonstration/test compartment for a second extinction test, which was designed to assess spontaneous recovery. This test session also terminated when a criterion of $5 \mathrm{~min}$ without responding had been reached.

The procedure was identical for Group Different, except that, prior to testing, each rat observed a demonstrator pushing the joystick in the direction that had not been reinforced during observer pretraining. Before being tested in the same way as the other observers, the rats in Group None were placed in the observation compartment for $10 \mathrm{~min}$, in which the house lights were on and the demonstration/test compartment, from which the joystick had been removed, was occupied by a demonstrator. This interval was chosen because it was anticipated, on the basis of training data, that it would take the demonstrators for Groups Same and Different an average of $10 \mathrm{~min}$ to make 50 responses while being observed.

\section{RESULTS}

One observer, assigned to Group None, was excluded from the experiment because it failed to make 50 reinforced responses during each of the final two pretraining sessions. Test data for 2 observers (one in Group Different and one in Group None) were lost because of an apparatus fault. Thus, for the purposes of analysis, the sample sizes were: Group Same $=8$, Group Different $=7$, Group None $=6$.

The demonstrators for Group Same took an average of $8.38 \min (S E M=1.63)$ to make 50 responses while being observed, and the demonstrators for Group Different took an average of $7.21 \mathrm{~min}(S E M=1.05)$. In 12 out of 15 cases, the demonstrators showed perfect discrimination while being observed, and in each of the remainder, just one response was made in the direction that had not been reinforced during demonstrator training.

Resistance to extinction was measured in both tests according to the number of previously reinforced responses that an observer made before reaching criterion. Previously reinforced responses were those that had been rewarded, for that observer, during discriminative pretraining. To find out whether observation training affected the direction of responding on test, observer performance was also measured in terms of the number of previously nonreinforced responses made before reaching criterion.
The data relating to each measure were subjected to analysis of variance in which group (same, different, none) and direction of pretraining (left, right) were factors. Where appropriate, this was followed by post hoc Newman-Keuls multiple-range tests comparing each Group with each of the others. Wherever, in the report that follows, Group Same and/or Different and/or None are said to have differed from one another, these tests revealed differences reliable at the $5 \%$ level. The data were also subjected to analysis of covariance, using percentage of nonreinforced responses during the first three sessions of pretraining and response rate during the last two sessions of pretraining as the covariates. In no case did either covary significantly with measures of test performance.

\section{Previously Reinforced Responding}

Figure 1 shows the mean number of previously reinforced responses to criterion for each of the groups, in Tests 1 and 2. In Test 1, Group Same made fewer previously reinforced responses to criterion than did Group Different, and Group Different made fewer than did Group None $[F(2,15)=16.91, p=.0001]$. In Test 2, Group Same made fewer previously reinforced responses to criterion than did Group None, whereas Group Different did not differ reliably from either of the other two groups $[F(2,15)=4.53, p=.029]$.

The analysis of Test 1 data also indicated that observers pretrained to push left $(M=32.00, S E M=5.53)$ made fewer previously reinforced responses to criterion than did observers pretrained to push right $(M=40.27, S E M=$ 8.06) $[F(1,15)=5.42, p=.034]$, but there was no group $X$ direction interaction $[F(2,15)=0.84]$. Rats tend to show a bias toward left responding in the apparatus used in this experiment, and it was thought that the effect of direction on previously reinforced responding might be due to Group Right's having received more partial reinforcement during pretraining. This hypothesis was sup-

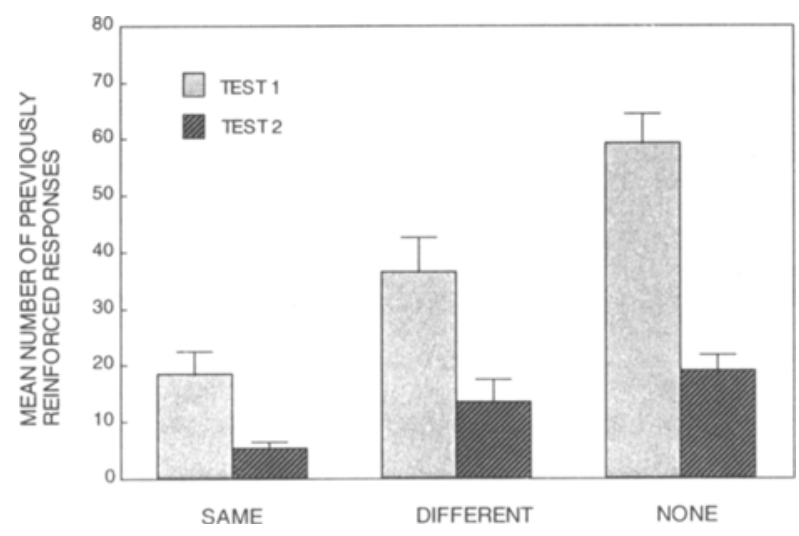

Figure 1. Mean number of responses that were reinforced during observer pretraining (previously reinforced responses) made by rats in Groups Same, Different, and None, in Tests 1 and 2. (Vertical lines indicate standard errors of the mean.) 


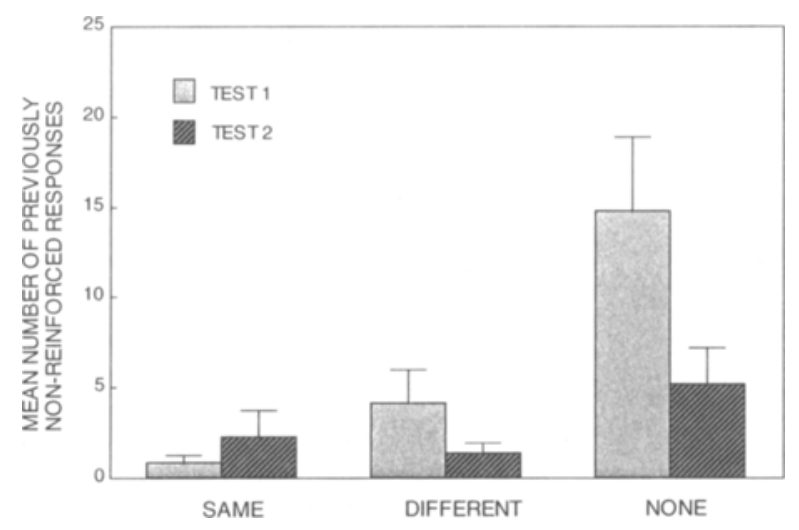

Figure 2. Mean number of responses that were not reinforced during observer pretraining (previously nonreinforced responses) made by rats in Groups Same, Different, and None, in Tests 1 and 2. (Vertical lines indicate standard errors of the mean.)

ported by an analysis of the pretraining data, which indicated that Group Right $(M=10.37, S E M=2.11)$ made a higher percentage of nonreinforced responses than did Group Left $(M=32.51, S E M=6.17)$ during the first three sessions of discrimination pretraining $[F(1,17)=$ $5.65, p=.030]$. Analysis of covariance, with percentage of nonreinforced responses during pretraining as the covariate and number of previously reinforced responses to criterion as the variate, indicated a reliable effect of Group $[F(2,14)=15.88, p=.0003]$, but no effect of direction $[F(1,14)=3.57]$ or of the covariate $[F(1,14)=0.07]$.

\section{Previously Nonreinforced Responding}

Figure 2 shows the mean number of previously nonreinforced responses to criterion for each of the groups, in Tests 1 and 2. In Test 1, Group Same and Group Different, which did not differ from one another, each made fewer previously nonreinforced responses to criterion than did Group None $[F(2,15)=8.89, p=.003]$. Groups Same, Different, and None did not differ reliably in the number of previously nonreinforced responses that they made to criterion in Test 2.

\section{DISCUSSION}

In the extinction test immediately after conspecific observation (Test 1), rats that had observed responding without reinforcement in the direction that had been reinforced during observer pretraining (Group Same) showed less resistance to extinction (i.e., extinguished faster) than did rats that had observed responding without reinforcement in the opposite direction (Group Different), and each of these groups showed less resistance to extinction than did animals that had been confined in the apparatus for a comparable period with a passive conspecific (Group None). Also in Test 1, rats that had observed a conspecific responding without reinforcement prior to testing (Groups
Same and Different) made fewer responses in the direction that had not been reinforced during observer pretraining than did rats that had been confined with a passive conspecific (Group None). In the extinction test $30 \mathrm{~min}$ later (Test 2), there was some evidence that rats which had observed a demonstrator pushing the joystick in the direction that had been reinforced during observer pretraining (Group Same) showed less resistance to extinction/spontaneous recovery than did rats that had been confined with a passive conspecific prior to Test 1 (Group None).

The difference between Groups Same and Different in Test 1 clearly indicates that prior observation of nonreinforced responding on the part of a conspecific can affect resistance to extinction in rats.

Demonstrators were observed responding without reinforcement, and observation of same responding resulted in less resistance to extinction than did observation of different responding. In a previous experiment (Heyes \& Dawson, 1990), when rats were allowed to observe demonstrators making reinforced responses, a contrary effect was found: observers of same responding were more resistant to extinction than observers of different responding. Although one must be cautious in drawing conclusions from comparisons across experiments, this contrast implies that observation of reinforced and nonreinforced responding have different effects on extinction in rats.

Inhibitory conditioning, learning of a response-noreinforcer relationship, by observation (Mackintosh, 1974), may have contributed to making Group Same less resistant to extinction than Group Different, and Group Different less resistant than Group None in Test 1. Such an account assumes that pretraining resulted in observers' expecting responses in one direction to be reinforced, and responses in the other direction to be nonreinforced, as well as that the observers in Groups Same and Different somehow equated the demonstrators' responses with their own. If this were the case, the rats in Group Same, which saw previously reinforced responses made in extinction, would have been subject to more expectancy disconfirmation, and would therefore have been more likely to learn a response-no-reinforcer relationship, than the rats in Group Different, which saw previously nonreinforced responses made in extinction. Furthermore, if there was some generalization between right and left responding, the rats in Group Different may have been more surprised, and therefore more likely to learn a response-noreinforcer relationship, than those in Group None, which did not observe responding in either direction prior to the test.

The results of Test 2 indicated that Group Same showed less spontaneous recovery than did Group None. If spontaneous recovery of an instrumental response is assumed to result from proactive interference (Mackintosh, 1974), this difference suggests that a response-reinforcement association acquired during pretraining interfered less in Group Same than in Group None with a subsequently 
learned response-no-reinforcement association. The rats in these two groups had identical pretraining and could therefore be expected to have acquired equally strong response-reinforcer associations. Consequently, the occurrence of less spontaneous recovery in Group Same implies that the members of that group had acquired, as a result of combined observational and direct extinction experience, a stronger response-no-reinforcement association than had the rats in Group None, which had only direct experience of extinction.

Both Group Same and Group Different made fewer previously nonreinforced responses than did Group None in Test 1 . If this were due solely to inhibitory learning by observation on the part of rats in Groups Same and Different, one might expect Group Different to have made fewer previously nonreinforced responses than Group Same. Group Different observed demonstrators making previously nonreinforced responses in extinction, whereas Group Same observed demonstrators making previously reinforced responses in extinction. The failure to find a difference between Groups Same and Different may have been due to a combination of generalization of inhibitory learning in Group Same, and imitative responding in Group Different.

Whatever the mechanisms involved, the results of the present experiment provide evidence of a new phenomenon, observational extinction. This phenomenon is distinguishable both from other examples of observational learning, in which observation of reinforced responding promotes response acquisition (e.g., Heyes \& Dawson, 1990; Heyes et al., 1992; Galef et al., 1986), and from latent extinction, in which exposure to the training context in the absence of reinforcement reduces resistance to extinction (e.g., Clifford, 1964; Robinson \& Capaldi, 1958; Seward \& Levy, 1949). The present experiment showed, in contrast, that observation of nonreinforced responding can reduce resistance to extinction in rats.

\section{REFERENCES}

Cufford, T. (1964). Extinction following continuous reward and latent extinction. Journal of Experimental Psychology, 68, 456-465.
GALEF, B. G. (1988). Imitation in animals: History, definition and interpretation of data from the psychological laboratory. In T. R. Zentall \& B. G. Galef (Eds.), Social learning: Psychological and biological perspectives (pp. 3-28). Hillsdale, NJ: Erlbaum.

GAlef, B. G., MANZjG, L. A., \& FieLD, R. M. (1986). Imitation learning in budgerigars: Dawson and Foss (1965) revisited. Behavioural Processes, 13, 191-202.

Henning, J. M., \& Zentall, T. R. (1981). Imitation, social facilitation, and the effects of ACTH 4-10 on rats ${ }^{\circ}$ bar-pressing behavior. American Journal of Psychology, 94, 125-134.

Heyes, C. M. (in press). Imitation, culture and cognition. Animal Behaviour.

Heyes, C. M., \& DAwson, G. R. (1990). A demonstration of observational learning using a bidirectional control. Quarterly Journal of Experimental Psychology, 42B, 59-71.

Heyes, C. M., DAwsON, G. R., NoKes, T. (1992). Imitation in rats: Initial responding and transfer evidence. Quarterly Joumal of Experimental Psychology, 45B, 229-240.

Heyes, C. M., Jaldow, E., Nokes, T., \& Dawson, G. R. (1993). Imitation in rats: The role of demonstrator action. Manuscript submitted for publication.

Hogan, D. E. (1986). Observational learning of a conditional hue discrimination in pigeons, Learning \& Motivation, 17, 40-58.

HoGAN, D. E. (1988). Learned imitation by pigeons. In T. R. Zentall \& B. G. Galef (Eds.), Social learning: Psychological and biological perspectives (pp. 225-238). Hillsdale, NJ: Erlbaum.

LEVINE, J. M., ZENTALL, T. R. (1974). Effect of a conspecific's presence on deprived rats' performance: Social facilitation vs distraction/imitation. Animal Learning \& Behavior, 2, 119-122.

MACKInTOSH, N. J. (1974). The psychology of animal learning. London: Academic Press.

Robinson, D. E., \& CaPALDi, E. J. (1958). Spontaneous recovery following non-response extinction. Journal of Comparative \& Physiological Psychology, 51, 644-646.

Roder, E. L., Timmermans, J. A., Vossen, J. M. H. (1989). The role of modelling in prevention and extinction of phobic behaviour in cynomolgus monkeys. Behaviour Research \& Therapy, 27, 637-645.

SewARD, J. P., \& LEVY, H. (1949). Latent extinction: Sign learning as a factor in extinction. Jourmal of Experimental Psychology, 39, 660-668.

THORPE, W. H. (1956). Learning and instinct in animals. London: Methuen.

Zentall, T. R., \& Levine, J. M. (1972). Observational learning and social facilitation in the rat. Science, 178, 1220-1221.
(Manuscript received October 28, 1992; revision accepted for publication January 26, 1993.) 